\title{
Inductively Coupled Electrical Stimulation - Part I: Overview and First Observations
}

\author{
Robert G Dennis, Ph.D.1 \\ 1 - Corresponding Author; Associate Professor of Biomedical Engineering, University of North Carolina \\ at Chapel Hill and NC State University; Owner, Micro-Pulse LLC, Chapel Hill, NC.
}

\section{Abstract}

\section{OPEN ACCESS}

Citation: Dennis R. (2019) Inductively Coupled Electrical Stimulation Part I: Overview and First Observations. ProcACIM 1(1): 20-35.

Editor: C. Norman Shealy M.D., United States

Received: March 11, 2019

Accepted: April 4, 2019

Published: July 2, 2019

Copyright: (c) 2019 ProcACIM. This is an open access article distributed under the terms of the Creative Commons Attribution License, which permits unrestricted use, distribution, and reproduction in any medium, provided the original author and source are credited.

Data Availability Statement: All relevant data are within the paper and its Supporting Information files.

Acknowledgements: DARPA contract number: N00173-01-1$\mathrm{G020}$, as a pilot project in the DARPA Metabolic Engineering Program, 2001 - 2002. Research since 2003 has been conducted using both personal and private funds and facilities.
Inductively Coupled Electrical Stimulation (abbreviated ICES) is a reliable, effective, non-invasive, pain-free, low cost, low-power and energy efficient method to apply micro-, nano-, and pico-ampere electrical stimulation to deep tissues. The design intent of ICES is to use magnetic pulses of a specific trapezoidal waveform to induce micro-, nano-, and pico-currents in tissues by electromagnetic induction rather than electrical conduction. Induction allows electromagnetic energy to pass through dry, intact, non-conductive garments, bandages, and tissues such as skin, to reach deep tissues and lesions with much higher energetic efficiency and volumetric uniformity than is possible using conductive energy transfer through the skin, thus conferring many advantages over conduction-based technologies such as TENS and micro-current stimulation devices. This is the first in a series of reports identifying the key electromagnetic parameters for inductively coupled electrical stimulation to yield consistent and reliable biological results. Details of the experimental PEMF apparatus used in the initial NASA studies, published in 2003 are reported, and for the first time the electro-magnetic methods are reported, analyzed in detail, and the observed biological effects of different waveform shapes are reported. To correct the omissions in the original NASA publication, the visible biological response observations are included, with the identification of the key electromagnetic parameters that were found to yield significant and repeatable biological effects. This paper is submitted in conjunction with a Letter to the Editor of this journal, which attempts to explain the reasons for the confusion and controversy surrounding PEMF research, and why it has proven so difficult to make scientific progress in the study of the effects of these apparently non-harmful, noninvasive electro-magnetic pulses on biological systems. The work reported in this paper is a first attempt to correct past errors and omissions, in line with the suggestions proposed in the Letter to the Editor.

\section{Introduction}

The observation that electro-magnetism has important biological effects is not at all an extraordinary claim. Visible sunlight and the adjacent ranges on the electromagnetic spectrum of infra-red and ultraviolet serve as the source of power for photosynthesis, allow vision, have many impacts on biological processes such as circadian rhythm, the production of vital substances such as vitamin D in the skin, and the transfer of warmth in the form of infra-red radiation, among many other 
known effects. Such a narrow slice of the electro-magnetic spectrum is only a diminishingly small fraction of the full range of this fundamental force of nature. To suppose that the full range of electro-magnetism could have biological effects aside from these that are known is not an extraordinary claim. It is a conservatively stated claim much more likely to be true than false. Therefore, investigations of the effects of pulsed electro-magnetic fields (PEMF) on biology should not be viewed with excess skepticism. These investigations should be subject to the same standards of proof and verification as any other field of study. Upon review of the scientific literature, and after discussing PEMF with clinicians who have experience with this treatment modality, one would be inclined to think that PEMF should already be a widely accepted, mainstream treatment modality for dozens of medical conditions that are very serious and are otherwise difficult or impossible to treat. And it should be noted that several PEMF technologies have the imprimatur of the US FDA, having been approved as safe and effective for more than one medical condition. Yet PEMF remains on the fringes of alternative medicine, and despite more than a thousand scientific and clinical reports of the positive and beneficial effects of PEMF, the field remains professionally stigmatized and marginalized. Any reasonable person would rightly ask why this is so.

The reasons for this are discussed in a Letter to the Editor of this journal (1), which describes some of the most serious challenges to and deficiencies in PEMF research, including the failure to publish negative results (publication bias), the tacit assumption that PEMF is "one thing" and thus it either works or it does not, and that the key parameters of PEMF are incorrectly assumed to be "frequency" and "Gauss". Upon detailed scrutiny of the published literature it becomes clear that most scientific reports on PEMF do not include sufficient detail on the PEMF methods or apparatus used in the study to allow independent replication, so the vast majority of peer-reviewed papers on PEMF do not meet the basic scientific requirement of reproducibility. Thus, these papers do not contribute to the scientific understanding of PEMF. As a result of these systematic deficiencies in the PEMF literature, much of the professional incredulity and marginalization of PEMF as a serious scholarly endeavor are self-inflicted wounds. These serious deficiencies need to be addressed by the PEMF researcher as they plan, conduct, and report their research.

For the purposes of this paper, the terms TVEMF and PEMF are equivalent, PEMF being the term that has enjoyed more widespread use in recent years, whereas the term TVEMF, more commonly in use when the NASA-JSC studies were carried out in the late 1990's, is retained for historical reference. The focus of this work is on a specific type of PEMF termed Inductively Coupled Electrical Stimulation, or ICES. This a specific form of pulsed electro-magnetic energy based upon a concept first reported in 1974 (2) and subsequently developed under contract for NASA-JSC in 1996 to 1999, then under contract for DARPA, and thereafter has been further refined using independent funding.

At NASA in 2003, TVEMF research was reported using "normal human neuronal progenitor" (NHNP) cells, which were cultured and exposed to five different electromagnetic waveforms, each applied at $10 \mathrm{~Hz}$ (3). Early gene array technology and 
standard cell culture assays were subsequently employed to determine the biological effects of only one of the five initially tested electro-magnetic waveforms in the study. The selected waveform, "square waves", were found to cause significant alterations in the expression of classes of genes, and metabolic function, cell and colony morphology by both light and electron microscopy, as described earlier (3). Crucially, however, the NASA report did not disclose the methods of the electro-magnetic pulse generator employed (designed and built by the author as an external consultant under contract with NASA), nor did the report disclose the findings on the different biological effects of each of the remaining different waveforms. The report published by NASA therefore has the key deficiency of failure to publish the negative results that were an important part of the study. However, this incomplete study has subsequently formed the basis for many of the commercially-available PEMF systems on the market today, which often incorporate the initial, unpublished findings from this study gleaned from informal technical discussions and during licensing and technology transfer negotiations. This previously unpublished information will be included and analyzed in this report as Experimental Series \#1. These observations were expanded and verified by studies performed at the University of Michigan using different cells and culture techniques. The unpublished findings from these initial studies have given rise to a series of subsequent experiments, with the specific objective of elucidating the waveforms responsible for the observed biological effects, first on cells grown in culture, then later upon tissues in living mammals.

\section{Research Design}

Initial experiments were to be conducted at NASA-JSC (NASA Johnson Space Center, Houston Texas) to validate the TVEMF system. First, it should be noted that electro-magnetic fields can be generated using a wide range of physical apparatus, including but not limited to coils, plates, and antenna. Scientists at NASA requested that the electro-magnetic fields be produced on the surfaces of flat electrical conductors, to facilitate future incorporation into bioreactors intended for use in earth orbit.

The main challenge was to identify a form of TVEMF that would have consistent beneficial biological effects. The scientists at NASA simply wanted to identify a form of TVEMF that facilitated cell growth and extracellular matrix (ECM) formation for use in experiments in the microgravity environment in orbit. So, as a consultant, the author of this paper undertook to identify a form of TVEMF that would facilitate cell growth and ECM formation using protocols and apparatus optimized for use on the Space Shuttle. After a thorough review of the extant PEMF literature and related studies including electrical tissue stimulation, which amounted to over 600 published papers, it became clear that the number of possible TVEMF/PEMF protocols were extremely numerous. In addition to "frequency" and amplitude, the parameters include the specific waveform (pulse) shape, as well as how the pulses are sequenced in a stimulation protocol, and also includes allowable tolerances in each parameter. 
By calculating the possible number of PEMF protocols on a spreadsheet, the resulting estimate was that there are approximately one quadrillion

$\left(1,000,000,000,000,000\right.$ or $\left.10^{15}\right)$ distinct PEMF protocols to choose from. Selecting from this large number at random would not be productive. To reduce this number to a tractable quantity for experimental purposes, several decisions were made a priori. The scientific decision was taken to use a pulse frequency of $10 \mathrm{~Hz}$, because this frequency had been reported numerous times to have desirable biological effects. The technical decision was made to strictly limit peak magnetic field strength and system power so as to facilitate usability of the system in the mid-deck lockers of the NASA Space Shuttles, which had strict technical requirements for flight hardware. This left open the remaining question of waveform shape.

The PEMF/TVEMF protocols reported in the available scientific literature on electromagnetic effects were categorized by the basic waveform shape that was employed: DC (direct current or fixed magnetism), sinusoidal, triangular, saw tooth, or square (Fig. 1, A-E). To this list was added very narrow pulses, called "delta pulses" (Fig. 1, F), which are employed in electrical engineering when a sharp edge dominates the effects on a system over the effects of a steady signal. Basically, a delta function isolates the edges of the "square wave", eliminating the steady plateau of each square wave from the waveform and focusing only on the effects of the sharp edges, both up and down. This classification scheme resulted in only six possible basic waveforms to consider, which is experimentally tractable. Holding all other variables constant to the extent possible, and using a continuous train of "10 $\mathrm{Hz}$ " pulses at the request of NASA (except for DC, which was steady), it became possible to build electro-magnetic pulse generators that were practically achievable and scientifically testable. Finally, due to the similarity of the triangle and saw tooth waveforms (Fig. 1, C and D), the saw tooth waveform was removed from the final list of waveforms to be tested initially. By this means, the very large number of experimental possibilities were reduced to only 5 distinct and well-defined experimental groups to be tested at NASA-JSC. 

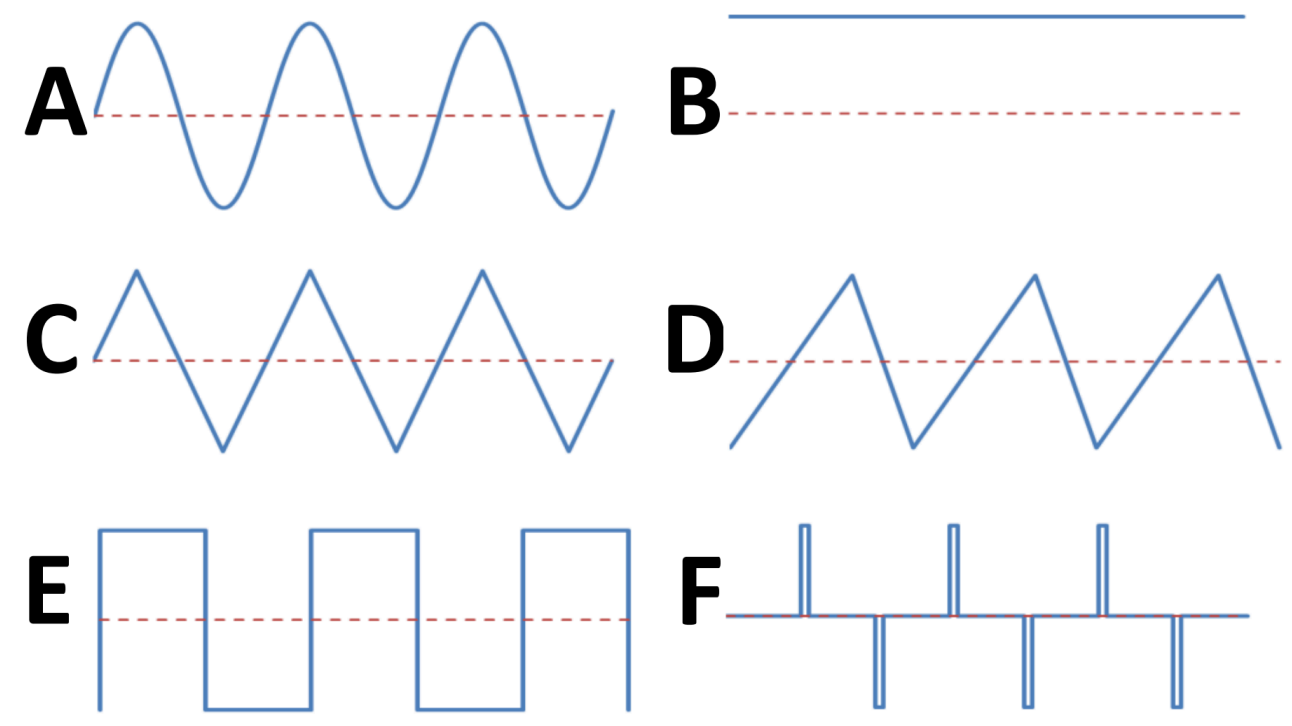

Figure 1: TVEMF Waveforms originally selected for testing at NASA-JSC: A sinusoid, B "DC" steady fixed magnetic field, C triangle, D saw tooth, which is a skewed or asymmetric triangle, E square wave, F delta pulse. For Experimental Series \#1, all pulses were delivered at $10 \mathrm{~Hz}$ (except the DC, which was not pulsed). In these diagrams, the dashed (red) line represents zero electromagnetism and the thick (blue) line represents the electromagnetic field applied to the cells in culture. Thus, the applied fields alternated symmetrically between positive and negative at $10 \mathrm{~Hz}$ (except DC, which was always positive).

The hypothesis to be tested was that certain PEMF waveforms would be more biologically effective than others when holding frequency and amplitude constant, and that these effects could be observed visually by grading the cell colony and ECM density in culture. Thus, the first experiment would use simple measures of cell colony growth, density, and morphology to assess the relative effectiveness of the waveforms under comparison. Subsequent molecular-genetic experiments at NASA were conducted using the most effective of these waveforms (square waves), but the important initial findings upon which that decision was made were never reported, and are given in detail here.

\section{Overview of Experiments}

The first series of experiments was carried out at the cell culture facilities at NASA Johnson Space Center, Houston TX (NASA-JSC). The NASA scientists referred to the pulsed electro-magnetism as "TimeVarying Electro-Magnetic Fields", or TVEMF. To verify and elaborate upon the first series of experiments done at NASA-JSC, a second series of experiments described below was conducted at the University of Michigan, in the laboratory facilities of the author, using different cell lines and functionally identical apparatus for applying electro-magnetic fields. If similar results were obtained, it would be more likely that the applied electro-magnetic pulses had 
general and repeatable effects over a relatively wide range of experimental conditions.

Experimental Series \#1: Two different electro-magnetic pulse generators were designed and built to supply the specific waveforms listed above. Electro-magnetic pulse generator 1 was constructed using a MAX038 High Frequency Waveform generator. This pulse generator was built as specified in the product datasheet (4) and configured to provide DC steady, sinusoidal, triangle, and square pulses at 10 $\mathrm{Hz}$. Pulse voltage and current were amplified using Operational Amplifiers and the gain was adjusted manually using fixed carbon-core resistors to achieve the desired magnetic field strength described below. This pulse generator is shown in the original NASA paper (3), Figure 1, in the blue and aluminum box on the shelf to the left of the open incubator. Throughout culture, pulses were applied continuously at $10 \mathrm{~Hz}$, or steadily in the case of DC.

Electro-magnetic pulse generator 2 was identical to a design reported earlier for use with the direct electrical stimulation of engineered and denervated tissues (5-10) to generate bipolar delta pulses at $10 \mathrm{~Hz}$. Each pulse was 200 microseconds in duration. These small pulse generators could be placed directly inside the incubator, near the culture dishes, because they had been originally designed for implantation into rats for neuromotor regeneration experiments.

Thin square platinum foil electrodes were used as conductors around which magnetic fields were produced when an electrical current was conducted from corner to opposite corner of the square electrodes. The electrodes were constructed of platinum metal foil, $.1 \mathrm{~mm}$ thick and $25 \mathrm{~mm}$ length on each edge. The electrodes were biocompatible so they could be placed either within the culture dish or below the culture dish as shown in the NASA paper, with wire soldered to opposite corners, as shown in Fig. 2. This configuration was selected because thin flat conductors generate wider and flatter magnetic fields across the space near the flat conductor. While the magnetic field strength near the flat conductor will be lower than the field at a similar distance from a round wire conductor, the thin flat conductor has the experimental advantages that the magnetic field varies less sharply with distance from the surface, so the cells in culture will be subjected to more uniform fields over a much larger area and depth than if a thin round conductor such as a wire were used. 


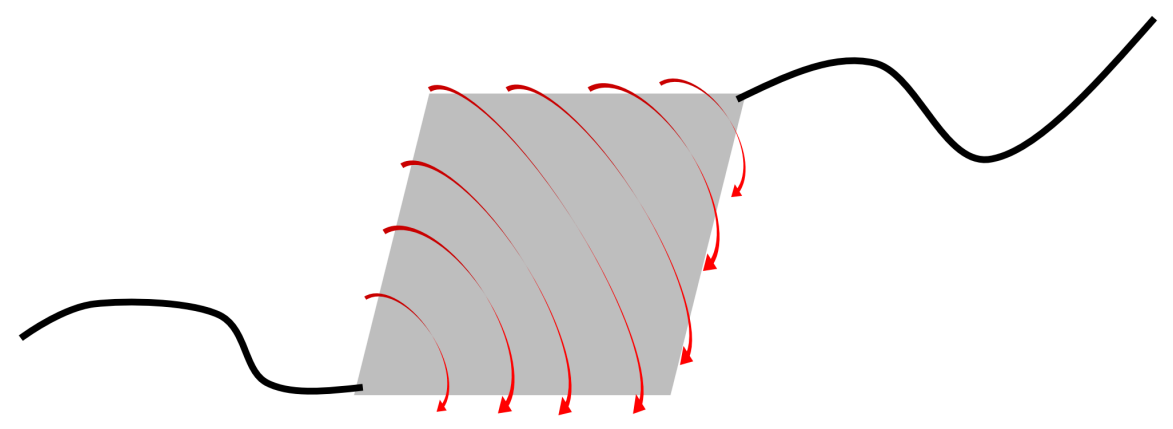

Figure 2: Square electrodes and magnetic fields around square electrode plates (NASA). The electrodes were energized by electrical current to generate magnetic fields around the square electrodes as shown by the red curved arrows. 
Results of Experiment Series \#1. Under the microscope it was clear that some colonies of cells subjected to TVEMF proliferated and expanded vigorously within 24 hours, whereas colonies not subjected to TVEMF showed no such proliferation within 24 hours, as shown in the NASA paper (3) Figs $4-6$. When viewed with the naked eye, for cells subjected to square wave or delta pulse TVEMF, thick and well defined colonies of cells could be seen forming directly over the electrodes, as shown in the NASA paper (3) Fig 2.

Once the most effective waveform was determined (square wave), the remainder of the NASA study (3) was carried out as reported, using only the square wave TVEMF. Briefly, gene arrays were analyzed using Gene Discovery Arrays and analysis software (InCyte Genome Systems Inc., St. Louis, Missouri). Additionally, cell metabolic parameters were measured, and images from light and scanning electron microscopy were analyzed. While no significant metabolic changes were reported, significant changes in cell colony growth patterns and gene expression were shown to occur. Large classes of genes were upregulated or down-regulated in concert, as shown in the tabulated data in the NASA paper.

Not reported in the NASA paper was the relative effect of different waveform shapes as assessed by visual inspection of the cell density over the conducting electrodes during the preliminary study, which in retrospect was perhaps the most important finding of this initial work.

To determine the most effective waveform, visual scaling was used to assess the effects of different TVEMF waveforms on NHNP cells in culture. The visual scale is shown in Fig. 3. While it is arguably qualitative, the differences in colony density and shape were readily apparent to the unaided eye, and results within experimental groups were highly consistent, whereas differences between groups were also clearly evident. The visual scale illustrates how the colony appeared with respect to the underlying square electrode, indicated by the dashed squares. The scaling ranged from 5 , indicating a very dense colony with sharply delineated rectilinear boundaries exactly matching the $25 \mathrm{~mm}$ square electrode, through decreasing degrees of density and boundary coincidence with the electrodes, down to zero, indicating no visible indication of increased cell colony density over the electrode. 


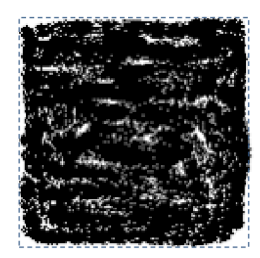

5

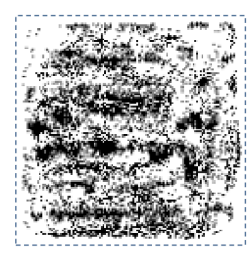

4

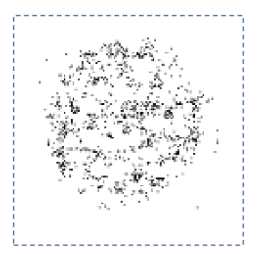

3

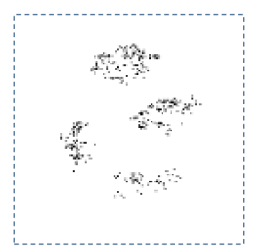

2

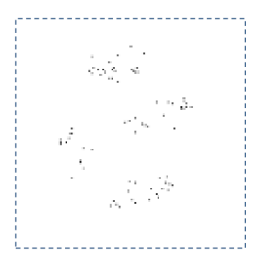

1

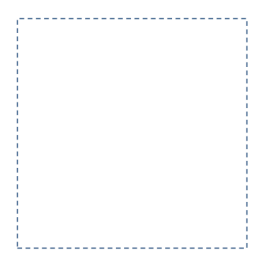

0

Figure 3: Visual scaling of the effects of different waveforms on NHNP cells in culture. The cells in culture would sometimes form dense colonies over the square electrodes when subjected to TVEMF. The qualitative visual scale illustrates how the colony appeared with respect to the underlying square electrode, indicated by the dashed squares. The qualitative scaling was based on the following: 5- dense colony with sharply delineated rectilinear boundaries exactly matching the $25 \mathrm{~mm}$ square electrode; 4- clearly visible colony with lower cell density and approximately square; 3 - single visible colony, approximately circular over the electrode; 2 - patches of smaller colonies over the electrode 543210 area; 1- hints of colonies over the electrode area (may just be random); 0no visible differences between TVEMF and control

Experimental Series \#2: After analyzing the preliminary results from the work done at NASA-JSC, a similar pilot study was conducted at the University of Michigan in the laboratory of the author (RG Dennis). This study was conducted for several purposes. First, to verify the findings from NASA-JSC using different cell lines; second, to expand the experimental groups to include a group with a strong DC magnet to provide a strong and steady magnetic field; and finally, to determine if the observations from the study at NASA could be generalized, or whether they were cell- or culture condition-specific.

In the U-Michigan study, two different skeletal muscle cell lines were used, one from primary explants from Sprague Dawley (rat) soleus muscle, and one from a commercially-established standard cell line (C2C12, from ATCC, Manassas, VA). Cell sourcing and culture were as described previously $(9,10)$. Skeletal muscle was selected because, like the nerve cells used at NASA, skeletal muscle is an electrically excitable tissue, and it was thought at the time that tissue excitability might be important for receptivity to the application of magnetic fields. Unlike the NASA-JSC study, this study also incorporated very strong solid magnets, rare earth neodymium cylinders of diameter $3 \mathrm{~mm}$ diameter and $10 \mathrm{~mm}$ length (kjmagnetics.com, catalog number D26). The rare earth element magnets were placed directly under the cell culture plates as the electrodes had been in the earlier pilot study at NASA-JSC. The resulting magnetic fields to which the cells in culture were subjected ranged from 1000 Gauss to 2500 Gauss. By comparison, the peak magnetic fields in the NASAJSC study generated by DC electric current through a flat conductor were designed to be at most $\sim 0.1$ Gauss.

Results of Experiment Series \#2. The key results from the second pilot study at UMichigan were identical to those from the NASA-JSC study, with the addition of the observation that even very strong solid magnets, with fields ten thousand times 
greater than those employed at NASA-JSC, still had no observable effect on cell colony formation or cell proliferation.

The work at NASA resulted in one paper (3) which reported only the detailed genetic and metabolic results for NHNC cells subjected only to $10 \mathrm{~Hz}$ square wave TVEMF, and two US patents (US 6485963 and US 6,673,597), which have been licensed from NASA for industrial use (royalty payments made from NASA to RG Dennis under NASA license \# DE-490, ALC 8000-0701). The pilot work done at the University of Michigan and subsequent privately-funded work was not published scientifically, but resulted in four additional US patents (US 8029432, US 8137258, US 8137259, and US 8376925) and two trademarks: ICES® and DigiCeutical®.

Summary of combined results from Experimental Series \#1 and \#2. Cell colony visual scaling observations from both studies are combined and tabulated in Table 1.

- When no electrical current was applied, no magnetic field was generated around the square conductor, and there was never any indication of a preference for cell colony growth or increased density over the inert square conductor under the culture dish.

- Steady DC magnetic fields, either small fields $(\sim 0.1 \mathrm{G})$ generated by steady electrical currents through the square conductor (at NASA) or strong magnetic fields (> $1000 \mathrm{G}$ ) from solid magnets (U-Michigan) never gave any visible indication of any effect on cell colony formation.

- Sine waves also gave no visual effects on cell colony formation or density.

- Triangle waves rarely, but not reliably, caused very slight increases in cell colony density over the square electrode, in a small number of cases.

- Delta Waves resulted in a very reliable increase cell density on the qualitative visual scale of 2 to 4 over the square conductor.

- Square waves caused a robust and reliable increase in cell colony density over the conductor, in many cases with the margin of dense colonies exactly matching the square shape of the underlying conductor.

The visual differences in colony formation fell into two very distinct and visually obvious groups:

(1) Virtually no detectable increase in colony density for control (no magnetic field), DC, sine and triangle experimental groups.

(2) Consistent and easily seen increases in cell colony density for both the delta wave and square wave groups. Qualitatively, the effects over the square wave were observed to be greater. The effectiveness of the delta waves was qualitatively estimated to be on the order of $70 \%$ to $80 \%$ as effective as the square waves. 


\begin{tabular}{c|c} 
Waveform & Scale \\
\hline None & 0 \\
\hline Steady & 0 \\
\hline Sine & 0 \\
\hline Triangle & $0-1$ \\
\hline Delta & $2-4$ \\
\hline Square & $3-5$
\end{tabular}

Table 1. Visual scaling of the effects of various TVEMF Waveforms on NHNP cell colony density and morphology in culture

\section{Discussion}

The key result from both initial studies is that magnetic fields that are steady (DC), or those that change smoothly (sine) or slowly (triangle) had no reliable, detectable effect on the growth rate or colony density of cells in culture, whereas rapidly changing magnetic fields had a reliable effect on cell proliferation and colony formation in culture so profound as to be readily visible by the unaided eye. This latter outcome was the case for both delta waves and square waves. It should be noted that the observable density of the colonies inside transparent cell culture dishes is due to the optical opacity of the cell colonies, which is a function of both the cell density (as reported in the NASA paper) as well as the nature and density of the extracellular matrix (ECM) produced by the cells. The production of ECM is an indicator of a cellular phenotype that facilitates tissue growth and repair. It was reported in the original NASA-JSC paper (3) that when studied in detail, the $10 \mathrm{~Hz}$ waveform resulted in up-regulation of families of genes involved in the production of ECM proteins.

Overall, the observations from these two pilot studies, both individually and taken together, demonstrate that reliable effects of applied magnetic fields on cell growth and colony formation in culture can only be expected under conditions where the magnetic field is switched on and off quickly. This is the case for both delta pulses and square waves, where the magnetic field changed sharply both at the beginning and at the end of each pulse. In the PEMF literature it is often incorrectly assumed that electro-magnetism can be switched on and off instantaneously. However, careful measurements of the magnetic field will show that perfect square waves are not achievable. In most cases the actual waveform is trapezoidal, as shown in Fig 4. The finite rate of change of a magnetic field over time is often referred to as the 
magnetic slew rate. This is simply the slope of the rising and falling edges shown in Fig. 4. The units for these slopes are reported in Gauss per second (G/s). For fields that change rapidly, the magnetic field slew rate can be reported in thousands of Gauss per second (kG/s) or millions of Gauss per second (MG/s). For comparison, the Earth's magnetic field is only about 0.5 Gauss, and it is relatively steady and unchanging over time, so its slew rate is essentially $0.0 \mathrm{G} / \mathrm{s}$.

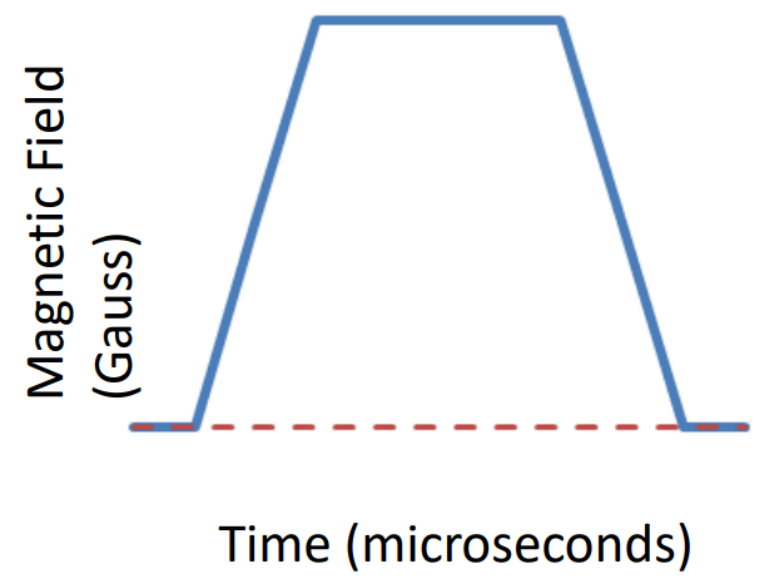

Figure 4: Trapezoidal Wave. All delta and square waves were actually trapezoidal waves with a finite slope (slew rate) of the magnetic field during the rising and falling edge of each pulse. The magnetic pulse slew rate was similar for all delta and square pulses in this study, so the primary difference between delta and square pulses was in the duration of each pulse, Delta pulses were relatively narrow, only 200 microseconds (0.0002 s), whereas square pulses were 50 milliseconds $(0.05 \mathrm{~s})$ in duration.

The second study (U-Michigan) served also to verify that for the conditions tested, only rapidly changing magnetic fields have reliable effects on cells in culture, that the static magnetic fields employed in the NASA-JSC study would have had no effect even if they had been thousands of times stronger, and that suitable electromagnetic waveform shapes will reliably result in noticeable effects that can be generalized to more than one set of cell culture conditions, including the use of different culture media and different cells (nerve and skeletal muscle), and the use of both primary cells and established cell lines.

Based on the observations of these studies, it becomes possible to evaluate the relative importance of various higher-level mechanisms of action to ask, what may be causing the visible effects?:

Thermal effects, the potentially growth-promoting effect of slightly warmer electrode surfaces under the culture dish, can be excluded because the steady DC magnetic field in the NASA study was generated by a steady (DC) electric current through the flat electrode, with an RMS current identical to the square wave in that same study, so the net heating of both electrodes would have been identical. However, the steady (DC) magnetic fields had no detectable effect on cell growth whereas the pulsed square wave fields had the maximum observed effects among 
all samples. Also, during device development the thermal heating of the electrodes was measured with current flowing, and no temperature change was detected because the Ohmic power dissipation through the electrodes was very small, by design.

Pure magnetic effects, those presumably due to the presence of any magnetic field, may also be excluded because steady (DC) magnetic fields had no detectable effects on cell growth and proliferation in culture, whereas identical much lower intensity magnetic field strengths, when pulsed at high slew rate, had consistent and readily detected effects all of the time, in every sample studied. Note that neither weak (DC current) nor strong (solid magnet) magnetic fields had any detectable effects on colony growth or density.

Slowly varying magnetic fields were also not effective. The sine and triangle waves were applied th the same peak magnetic field strength as the delta and square waves, but the smooth and slow magnetic field transitions had no reliably detectable biological effects, with only the smallest effects rarely observed from many dozens of culture dishes to which these smooth and slowly changing fields were applied. These barely detectable cell colony patterns were believed to represent only small random variations in the cell colonies in different dishes, and were not notably different from those in the control (no waveform) groups.

Effects primarily related to pulse frequency were also excluded. While the scientific literature reports that high frequency sine waves in the $\mathrm{MHz}$ (mega hertz) range are believed to have biological effects, the studies herein focused on very low frequency, specifically $10 \mathrm{~Hz}$. The sine and triangle waves in this series of experiments were applied at the same $10 \mathrm{~Hz}$ frequency as the delta and square waves, but only the delta and square waves had detectable effects on cell growth and colony formation. For this reason it is possible to exclude frequency as the primary parameter of importance for low-frequency PEMF as studied in these experiments.

Rapidly changing magnetic fields were the only waveforms that yielded highly reliable, repeatable, easily detected effects on cell colony formation and growth. These included both the delta wave and the square wave. In subsequent studies (3), only the square waveforms were used, and the resulting biological effects were later studied in more detail, indicating a range of effects that were correlated to very significant changes in gene expression.

\section{Post Analysis of Experimental Apparatus:}

Because it only became clear after these studies were concluded that only rapidly changing waveforms would induce biological effects, at the end of the studies a more detailed measurement was made of the waveforms generated by the pulse generators and electrodes used in these studies. The peak magnetic fields were measured to be about 0.1 Gauss, as expected, and the magnetic field slew rates were estimated to be in the range of $20 \mathrm{kG} / \mathrm{s}$ to $40 \mathrm{kG} / \mathrm{s}$ for the square wave, and exceeded $100 \mathrm{kG} / \mathrm{s}$ for the delta waves. The slew rate was measured for both the rising and falling edges of the trapezoidal pulses. The delta wave had a similar 
waveform shape but greater slew rate than the square wave pulses. The delta wave generators had stronger and higher-speed output drivers, so they were able to change the current through the flat square conductor more rapidly. These slew rate estimates were based on oscilloscope traces of electrical current flowing through each conductor during each pulse edge as a function of time, as a proxy measure for the resulting magnetic field. For comparison, the slew rate for triangle wave pulses would be $\sim 2 \mathrm{G} / \mathrm{s}$, which is many thousands of times slower than the slew rate for either the square or delta wave pulses.

The magnetic fields definitely did not change instantaneously for either pulse waveform, so it is more accurate to think of both the delta and the square pulses as trapezoidal pulses (Fig. 4) where they differ primarily in the width of the pulse (Fig. 2, E vs. F), and not in the critical parameter of magnetic field slew rate. Delta pulses, owing to their very narrow pulse width, convey much lower total electro-magnetic energy than square waves. Using the pulse width as an approximation for the area under each pulse, the relative energy can be calculated. Square waves were continuous, at $10 \mathrm{~Hz}$, and bipolar (swinging negative to positive and back), so each square pulse was $50 \mathrm{~ms}$ wide. By comparison, each delta pulse was set to only 200 microseconds pulse width. Because the pulses all had the same amplitude, the ratio of the pulse widths yields a good estimate of the relative electro-magnetic energy in each pulse:

$$
\begin{aligned}
& \text { Delta pulse width }=0.0002 \text { seconds } \\
& \text { Square pulse width }=0.05 \text { seconds }
\end{aligned}
$$

The energy ratio of the pulses $=$ delta/square $=0.0002 / 0.05=0.004(=0.4 \%)$ Stated another way, the delta pulses yielded very similar, albeit slightly smaller, biological effects, probably at least $80 \%$ of the biological effects of a continuous square wave, while only requiring $0.4 \%$ of the electro-magnetic energy to do so. Thus, a major margin of electro-magnetic safety and electrical efficiency may be acquired, by a factor of $\sim 250$ fold, or approximately a $99.6 \%$ reduction in electromagnetic energy, by simply employing narrow delta pulses rather than using continuous square wave pulses in clinical and research PEMF systems. Another way of looking at this finding is that at least $80 \%$, and probably much more, of the biological effect of low-frequency, low-intensity magnetic pulses seems to be attributable to the slew rate of the magnetic field intensity, and much less of the biological effect may be attributed to the steady magnetic component of each pulse.

\section{Conclusions}

This paper pulls together the pilot data from a number of experiments conducted at NASA-JSC and the University of Michigan under similar experimental conditions, using functionally identical electromagnetic TVEMF/PEMF equipment to apply pulses, allowing observations to be made regarding the effects of various electromagnetic waveforms on cells grown in culture.

Though not reported in the original NASA paper (3), these findings were strong enough to allow the NASA-JSC study to move forward with high reliability between 
groups, allowing a strong effect to be shown when $10 \mathrm{~Hz}$ square wave TVEMF was applied. In that paper, it was subsequently reported that the effects of TVEMF could be clearly detected at the level of gene expression, with TVEMF favoring the expression of whole classes of genes related to cell growth and tissue development.

These observations reinforce and elaborate upon the widely expressed belief, both clinical and scientific, that electro-magnetic energy in its differing waveforms, frequencies, and intensities can have the widest imaginable range of biological effects, ranging from those effects essential to life (such as sunlight and warmth), through a diverse continuum including effects that are beneficial to health (such as various forms of micro-current stimulation and PEMF), then on toward those forms of electro-magnetic radiation detrimental to health (such as electro-magnetic smog) all the way to forms that are randomly transformative, causing mutations, and lethal forms including ionizing radiation, pulses sufficient to electrocute or cause cardiac arrest, severe burns, or death. Considering low-frequency pulses $(10 \mathrm{~Hz})$ at very low intensity, we observed that the shape of the waveform itself is the major determinant of the observed biological effects. Based on these observations, it is reasonable to hypothesize that the beneficial effects of various forms of PEMF may be very different in their fundamental mechanisms of molecular and biophysical transduction, and thus might reasonably be expected to have differing clinical effects. PEMF as currently employed clinically, which spans a wide range of frequencies, waveforms, and intensities, should not be considered a single uniform clinical tool. The biological effects of differing forms of PEMF may depend at least as much on precise waveform shape as on any other parameter such as frequency.

The consistent finding that only rapidly changing magnetic fields had reliable and easily observed effects on cell growth and colony formation in culture should not be taken to mean that other forms of electromagnetism are necessarily ineffective, especially under very different circumstances. Cells in culture are notoriously arrested in their phenotype, lacking the complex structures and emergent properties of intact, adult-phenotype tissues (7-10). While we were unable to show any effects of static magnets on cell growth in culture, there are numerous reports of static magnets being used with success in the clinical treatment of bone and joint injuries (11). This is likely due to the fact that intact complex tissues are able to respond to magnetic fields in much more complex and nuanced ways than are individual cells in culture. So it would be incorrect to conclude from our data that specific forms of PEMF are necessarily ineffective. Extrapolating reported effects observed in cell cultures to effects expected in whole organisms should be done with great caution.

The deficiencies of these pilot studies are several in number, including the fact that they were entirely exploratory, so while each experiment was conducted with utmost care, the overall experimental design was not optimized to elucidate the effects eventually observed. The key initial observations were qualitative, and their importance was only recognized long after they had been collected. As a result, key parameters such as magnetic pulse slew rate were not known until after all studies were completed, and were not varied nor controlled to elucidate the effects of controlled variations in slew rate. In future studies we have corrected these 
deficiencies, and these effects have been studied in more detail and will be reported in subsequent papers.

\section{References:}

1. Dennis, R.G. Letter to the Editor: Why so much confusion about PEMF? JCIM, volume 1(1), 2018.

2. Bassett et al, Augmentation of bone repair by inductively coupled electromagnetic fields, Science, New Series, v. 184, No. 4136, pp. 575-77, May 3, 1974

3. Goodwin, T.J. Physiological and Molecular Genetic Effects of Time-Varying Electromagnetic Fields on Human Neuronal Cells. NASA/TP-2003-212054, 2003, available electronically from: https://ntrs.nasa.gov/archive/nasa/ casi.ntrs.nasa.gov/20030075722.pdf

4. https://pdfserv.maximintegrated.com/en/ds/MAX038.pdf

5. Dennis R.G. Bipolar implantable stimulator for long-term denervated muscle experiments. Med \& Biol Eng \& Comput, March, 36: 225-28, 1998.

6. Dennis, R.G., Dow, D.E., Faulkner, J.A. An implantable device for stimulation of denervated muscles in rats. Medical Engin \& Physics, 25(3), pp. 239-253, 2003.

7. Dennis, R.G., Dow, D. E. Excitability of skeletal muscle during development, denervation, and tissue culture. Tissue Engineering, 13:10, 2395-2404, Oct. 2007.

8. Kosnik P. Jr., Faulkner J.A., and Dennis R.G., Functional development of engineered skeletal muscle from adult and neonatal rats. Tissue Engineering 7(5) 573-584, 2001.

9. Dennis, R.G., Paul E. Kosnik, Mark E. Gilbert, and John A. Faulkner. Excitability and contractility of skeletal muscle engineered from primary cultures and cell lines. Am J Physiol Cell Physiol 280: C288-C295, 2001.

10. Dennis R.G., Kosnik P.E. Excitability and isometric contractile properties of mammalian skeletal muscle constructs engineered in vitro. In Vitro Cell. Dev. Biol. Anim. 36(5): 327-335, 2000

11. Efficacy of a Static Magnetic Device Against Knee Pain Associated with Inflammatory Arthritis JCR: Journal of Clinical Rheumatology: October 1999 Volume 5 - Issue 5 - ppg 302-304 